Notfall Rettungsmed 2022 $25: 174-176$

https://doi.org/10.1007/s10049-022-00993-2

Angenommen: 1. Februar 2022

Online publiziert: 28. Februar 2022

(c) The Author(s), under exclusive licence to Springer Medizin Verlag GmbH, ein Teil von Springer Nature 2022

\section{Prähospitale Reanimations- versorgung in Zeiten der Pandemie - ein erster Blick auf Über- und Unterversorgung im Alter}

Andreas Günther · Sybille Schmid

Feuerwehr, Stadt Braunschweig, Braunschweig, Deutschland
Seit Beginn der COVID-19-Pandemie liegen die Sterbefallzahlen in Deutschland teilweise über den mittleren Werten der Vorjahre. Diese Übersterblichkeit ist nicht nur auf COVID-19-Tote zurückzuführen. Besonders betroffen ist die Altersgruppe ab 65 Jahren [1]. Die Auswirkungen der Pandemie beeinträchtigen den Zugang zur Gesundheitsversorgung und betreffen auch die Reanimationsversorgung durch den Rettungsdienst. Allerdings scheinen sich deutschlandweit die Häufigkeit von prähospitalen Reanimationsversuchen pro Einwohner (CPR-Inzidenz) und die Altersstruktur der betroffenen Patienten während des Jahres 2020 im Vergleich zum Vorjahr nicht verändert zu haben [2].

Vor diesem Hintergrund wird aktuell untersucht, wie sich prähospitale Reanimationsversuche (CPR) in der Stadt Braunschweig entwickelt haben. Hierfür wurden Melde- und Reanimationsregisterdaten des Zeitraums vom 01.07.2011 bis zum 30.06.2021 in zehn Studienabschnitte, jeweils von Juni bis Juli, eingeteilt und retrospektiv ausgewertet. Im gesamten Studienzeitraum erfolgten durchschnittlich 206 CPR, wovon $14 \%$ zu einer Lebendentlassung führten. Bei durchschnittlich 249.572 Einwohnern (EW) lag die Sterberate bei 114 Sterbefällen pro $10.000 \mathrm{EW}$ pro Jahr.

\section{Patienten ab 65 Lebensjahren}

In der Studienregion war die deutschlandweite Übersterblichkeit während verschiedener Pandemiemonate mit den zwölfmonatigen Zeitabschnitten nicht nachweisbar. Auffällig ist ein Anstieg der Sterberate vor Beginn der Pandemie bei Menschen ab 65 Jahren. Im Studienabschnitt des Pandemiebeginns fiel die Sterberate und stieg dann wieder an. Diese Entwicklung ist in - Abb. 1 grafisch dargestellt. Da der untersuchte Versorgungsbereich bislang weniger von der Pandemie betroffen ist als Deutschland insgesamt, könnten die Auswirkungen auch während der höchsten Übersterblichkeit zum Jahresende 2020 hier geringer gewesen sein. Möglicherweise können Betrachtungen von anderen Zeitabschnitten Veränderungen der Sterberaten aufdecken, die mit den deutschlandweiten Daten korrelieren. Allerdings würden kürzere Abschnitte die ohnehin kleinen Fallzahlen der CPR reduzieren.

\section{Lebendentlassungen}

Bei Menschen ab 65 Jahren erfolgten durchschnittlich 141 CPR (Min. 119 - Max. 176; SD 19) pro Studienabschnitt. Das Alter dieser Patienten betrug 79 Jahre (65-99; 8), 42\% waren weiblich. Lebend entlassen wurden im Mittel 9\% (4-12; 3). - Abb. 1 verdeutlicht, dass der niedrigste Wert von $4 \%$ vor Pandemiebeginn lag. Im gleichen Studienabschnitt von Juli 2018 bis Juni 2019 erreichte auch die Sterberate ihren Höchstwert. Diese Auffälligkeit bedarf weiterer Untersuchungen. Ein Zusammenhang mit der Pandemie oder der Grippewelle 2017/18 ist zeitlich ausgeschlossen. Nach Beginn der Pandemie waren Lebendentlassungen wieder häu- 


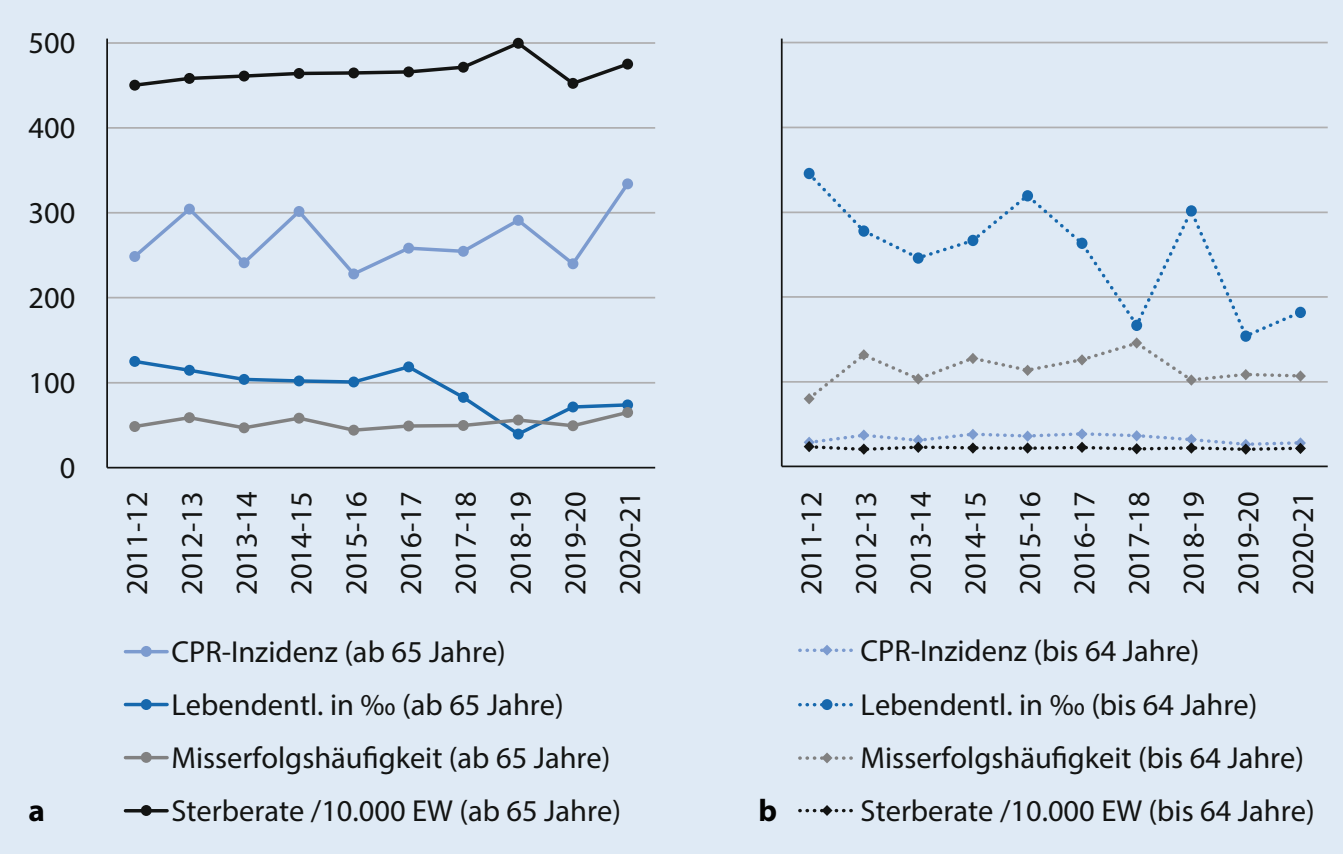

Abb. 14 Die Entwicklung
von Lebendentlassungs-
quote, CPR-Inzidenz, Miss-
erfolgshäufigkeit und Ster-
berate im Verlauf der Stu-
dienabschnitte bei Pati-
enten ab 65 und bis un-
ter 65 Jahren. Studienab-
schnitt 01.07. bis 30.06.
des Folgejahres; Reanima-
tionsversuch (CPR); Le-
bendentlassungsquote
(Lebendentl.); Einwoh-
ner (EW); CPR-Inzidenz
in CPR/100.000 EW/Jahr;
Misserfolgshäufigkeit in
CPR ohne Lebendentlas-
sung/1000 Sterbefälle/Jahr

figer, erreichten aber nicht das vorherige Niveau. Diese selteneren Lebendentlassungen sind gut mit den Ergebnissen der Referenzgruppe des Deutschen Reanimationsregisters aus 2020 vereinbar [2].

\section{CPR-Inzidenz}

Eine Perspektive auf Systemebene eröffnet die CPR-Inzidenz [3]. In der Altersgruppe ab 65 lag die CPR-Inzidenz bei 270 pro 100.000 EW jährlich (228-334; 35). Die Abbildung verdeutlicht die starken Schwankungen und den Höchststand im letzten Studienabschnitt. Dieser liegt vollständig in der Pandemie.

\section{Misserfolgshäufigkeit}

Die Relation von CPR zu Sterbefällen ermöglicht einen anderen Systemblick auf die Reanimationsversorgung [4]. Misserfolgshäufigkeit (MEH) wurde definiert als Anzahl der CPR mit Tod vor Krankenhausentlassung pro Sterbefall und Jahr. Bei Menschen ab 65 betrug die MEH 53 (44-65; 7) pro 1000 Sterbefälle jährlich. Den höchsten Wert erreichte die $\mathrm{MEH}$, wie die CPR-Inzidenz, im letzten Studienabschnitt.
Über- und Unterversorgung

Wiederbelebungsmaßnahmen sind nicht bei allen sterbenden Menschen indiziert. Sie werden nicht bei jedem Kreislaufstillstand durchgeführt. Eine Versorgung mit nicht indizierten Maßnahmen wird als Überversorgung bezeichnet. Unterversorgung besteht dagegen, wenn Maßnahmen indiziert sind, aber nicht erfolgen [3, 5].

\section{》) Die MEH zeigt, dass mehr Menschen an ihrem Lebensende einem vergeblichen Reanimations- versuch unterzogen wurden}

In der Altersgruppe ab 65 weist der Höchstwert der CPR-Inzidenz im letzten Studienabschnitt darauf hin, dass Unterversorgung abgenommen hat. Zusätzlich zeigt der Höchstwert der MEH im gleichen Zeitraum, dass mehr Menschen an ihrem Lebensende einem vergeblichen Reanimationsversuch unterzogen wurden. Folglich scheint die Balance zwischen Über- und Unterversorgung mit CPR in der Pandemie von Unter- zu Überversorgung verschoben. Dies ist die Perspektive der Systemebene. Aussagen für den konkreten Versorgungsfall sind nur bei einer individuellen Beurteilung möglich.

\section{Vergleich mit jüngeren Patienten}

Bei Menschen jünger als 65 Jahre erfolgten durchschnittlich 65 CPR $(52-76$; 9) pro Studienabschnitt. Das Alter dieser Patienten betrug 51 Jahre $(0-64 ; 12), 23 \%$ waren weiblich. Die Lebendentlassungen lagen bei $25 \%(15-35 ; 66)$. Die CPR-Inzidenz betrug 33 pro 100.000 EW jährlich $(26-38 ; 5)$ und die MEH 114 pro 1000 Sterbefälle pro Jahr (80-146; 19).

Die gemeinsame Betrachtung von CPRInzidenz und MEH über den gesamten Studienzeitraum zeigt, dass in der Altersgruppe unter 65 CPR seltener waren, aber Versterbende häufiger einer vergeblichen CPR unterzogen wurden. Im Vergleich zur Altersgruppe ab 65 ist die Balance zwischen Über- und Unterversorgung zur Überversorgung verschoben. Dies kann als Hinweis auf altersgerechten Zugang zur Reanimationsversorgung gewertet werden [4]. Bei unter 65-Jährigen waren Lebendentlassungen seit Beginn der Pandemie seltener. Dazu könnten unter anderem veränderte Vorgehensweisen bei der Reanimationsbehandlung beigetragen haben. Dieses Ergebnis ist gut mit publizierten Daten vereinbar [2]. 


\section{Fachnachrichten}

\section{Schlussfolgerungen}

Seit Beginn der Pandemie zeigen erste Auswertungen im untersuchten städtischen Versorgungsbereich keine Hinweise auf eine Zunahme der Unterversorgung mit prähospitalen Reanimationsversuchen bei Menschen ab 65 Jahren. Eine ergänzende Perspektive auf die Reanimationsversorgung bietet die $\mathrm{MEH}$ mit dem Bezug von CPR auf Sterbefälle. Die differenziertere Betrachtung von Überund Unterversorgung auf Systemebene weist auf eine Verschiebung der Balance zwischen Über- und Unterversorgung in Richtung Überversorgung hin. CPR nach prähospitalem Kreislaufstillstand führten seltener zu einer Lebendentlassung aus dem Krankenhaus. Dieses Resultat ist mit den Ergebnissen des Reanimationsregisters aus dem Jahr 2020 vereinbar.

\section{Korrespondenzadresse}

\section{Andreas Günther}

Feuerwehr, Stadt Braunschweig

Eisenbütteler Straße 2, 38122 Braunschweig,

Deutschland

andreas.guenther@braunschweig.de

Danksagung. Wir danken Hubert Philipp und Juliane Meyer vom Referat Stadtentwicklung und Statistik der Stadt Braunschweig für die Aufbereitung der Einwohner- und Sterbedaten.

\section{Einhaltung ethischer Richtlinien}

Interessenkonflikt. A. Günther und S. Schmid geben an, dass kein Interessenkonflikt besteht.

Für diesen Beitrag wurden von den Autoren keine Studien an Menschen oder Tieren durchgeführt. Für die aufgeführten Studien gelten die jeweils dort angegebenen ethischen Richtlinien.

\section{Literatur}

1. Statistisches Bundesamt (2021) Sterbefallzahlen und Übersterblichkeit. https://www.destatis.de/ DE/Themen/Querschnitt/Corona/Gesellschaft/ bevoelkerung-sterbefaelle.html. Zugegriffen: 6 . Dez. 2021

2. Fischer M, Wnent J, Graesner J et al (2021) Jahresbericht des Deutschen Reanimationsregisters. Außerklinische Reanimation 2020. Anästhesiologie Intensivmed 62:V68-V73

3. Gräsner JT, Herlitz J, Tjelmeland I et al (2021) Epidemiologie des Kreislaufstillstandes in Europa. Leitlinien des ERC 2021. Notfall Rettungsmed 24:346-366
4. Günther A, Harding U, Gietzelt M et al (2015) Ein städtischer Rettungsdienstbereich am Beginn eines sektorenübergreifenden Qualitätsmanagements. ZEFQ 109(9-10):714-724. https://doi.org/ 10.1016/j.zefq.2015.06.003

5. Scherer M, Wagner H-O, Lühmann D et al (2018) Schutz vor Über- und Unterversorgung. S2eLeitlinie. AWMF-Register-Nr. 053-045, DEGAMLeitlinie Nr. 21.DEGAM, Berlin

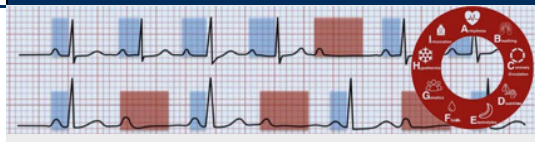

\section{Das ABC des EKGs}

Alle 9 Artikel digital und in Notfall+Rettungsmedizin

Die EKG-Befundung ist im notfallmedizinischen Alltag nicht wegzudenken. Als schnelle, günstige und nebenwirkungsfreie Methode können bei guter Interpretation bereits viele Informationen gewonnen werden. Erkennen auch Sie die typischen Muster, die auf lebensbedrohliche bzw. notfallmedizinisch relevante Erkrankungen hinweisen?

Diese Serie ermöglicht dem Leser graphisch eine schnelle Mustererkennung und vermittelt außerdem durch die strukturierte Sammlung der verschiedenen EKGBilder spannendes Wissen.

Die Serie führt systematisch durch die wichtigsten EKG-Veränderungen verursacht durch:

- Arrhythmia

- Breathing

- Coronary Circulation

- Disabilities

- Electrolytes

- Fluids

- Genetics

- Hypothermia

- Intoxication

Die komplette Serie mit praktischen Handlungsanweisungen für die Befundung und weiterführenden Erläuterungen lesen Sie digital gebündelt auf springermedizin.de.

Scannen Sie den QR-Code und finden Sie alle Artikel auf einer Seite.

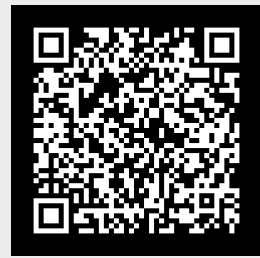

